\title{
Sujeción y Resistencia de Sujetos LGTB en la Educación Secundaria
}

\section{Subjection and Resistance LGTB Subjects in the Secondary Education}

\author{
Sylvia Contreras-Salinas ${ }^{1 *}$ \\ Mónica Ramírez-Pavelic ${ }^{2}$ \\ ${ }^{1}$ Universidad de Santiago de Chile ${ }^{2}$ Universidad Arturo Prat
}

\begin{abstract}
Este artículo expone algunos resultados de una investigación fenomenológica retrospectiva llevada a cabo en la isla Gran Canaria-España con sujetos que se identificaban como parte del colectivo LGTB. El objetivo establecido fue descubrir las definiciones que construyen de sus procesos de sujeción, resistencia y visibilización en los contextos escolares. La indagación utilizó como técnica la entrevista en profundidad que se aplicó a 26 participantes que narraron su paso por los escenarios educativos. Desde un pasado-presente se construyen relatos que desvelan los significados y estrategias que ponen en juego sujetos cuya singularidad es cuestionada, sancionada y excluida del espacio social educativo, apreciándose la educación secundaria como un escenario menos constreñido que la primaria, en que la pluralidad se hace presente, otorgando la posibilidad de prácticas de resistencia y sabotaje a los sujetos.
\end{abstract}

Descriptores: Diversidad sexual, Resistencia, Identidad, Educación.

This article presents some results of a retrospective phenomenological research carried out on the island Gran Canaria-Spain with individuals who identified themselves as part of the LGTB community. The stated objective was to discover the definitions that build their processes clamping strength and visibility in school contexts. The investigation used as in-depth interview technique was applied to 26 participants who narrated their way through educational settings. From a pastpresent stories that reveal the meanings and strategies put into play subjects whose uniqueness is questioned, punished and excluded from the educational social space are constructed, appreciating secondary education as a less constrained than the primary stage, wherein the plurality is present, the possibility of granting practices of resistance and sabotage subjects.

Keywords: Sexual diversity, Resistance, Identity, Education.

Este artículo se construyó en base a los resultados expuestos en un capítulo de tesis doctoral que está citada en las referencias bibliográficas.

*Contacto: sylvia.contreras.s@usach.cl

ISSN: 0718-7378

www.rinace.net/rlei/
Recibido: $\quad 18 / 07 / 2016$

$1^{\text {a }}$ Evaluación: 30/08/2016

Aceptado: $\quad$ 15/09/2016 


\section{Introducción}

La educación secundaria se sitúa en la actualidad en una posición que origina oportunidades, demandas y expectativas generalizadas, ubicándola en un lugar clave en la configuración societal, transfiriéndosele muchas veces los efectos de las crisis económicas, políticas y sociales que se dan cita en determinados periodos históricos. A partir de aquí, se convierte en una educación que se constituye en y desde la compleja necesidad de orientar la formación de adolescentes que se encuentran en proceso de transformarse en adultos y que incluye, además, los diversos significados que cada sociedad otorga a esta etapa vital (Cadafalch, 2001), cuyo eje estructurante se caracteriza por la incertidumbre y la vulnerabilidad social de sus trayectorias (Castel, 2006).

Por otra parte, se complejiza la función de este nivel educativo al confrontar un escenario de desconfianza creciente hacia la escuela, tanto por parte de la sociedad como de sus propios protagonistas. Desconfianza que se agudiza al constatar que se carece tanto de consenso político y social en materia educativa, como de estrategias en la formación inicial del profesorado de secundaria, lo cual impide dar respuesta a las demandas de los jóvenes, atisbándose tensiones que giran permanentemente alrededor de la cultura juvenil y que no se resuelve con medidas simples o unidimensionales (UNICEF, 2010).

A partir de lo anterior, es innegable el deficiente funcionamiento del sistema educativo a este nivel, el cual se refleja principalmente en los altos niveles de fracaso y abandono escolar (Vicente, Galiano y Miranda, 2015). Asimismo, en un marco en que la oferta de la educación secundaria es la que determina el nivel educativo que alcanzará la mayoría de la población, es esperable que desde sus aulas se defina gran parte del perfil cultural, económico y ciudadano de las sociedades (UNICEF, 2010). En este contexto, la presión que se desencadena en dichos espacios podría deberse a la alta intromisión del adulto-docente que es vivida generalmente como autoritarismo o por su nula mediación sentida como "dejar hacer", siendo innegable la tensión entre los estamentos juvenil y adulto que se transmite a través de la cultura escolar. A partir de aquí, se origina en muchos casos una relación antagónica por considerar que existen diferencias irreconciliables entre ambos.

Desprendiéndose la relevancia que adquiere la escuela secundaria en la formación, con miras al porvenir que los jóvenes vislumbran para sí mismos en el marco de sus particulares condiciones de significación y agencialidad (D'Aloisio, 2015), puesto que este espacio social y sus mecanismos de exclusión/inclusión tienen efecto en la configuración de sus subjetividades, lo que resulta relevante si consideramos que los escenarios educativos secundarios se configuran en esta lógica, tal como plantea Bernstein (1994) al señalar que la concreción de estrategias culturales interpretativas comporta categorías impuestas diferencialmente por los principios de clasificación.

De este modo, los escenarios educativos secundarios en comparación con los primarios, son vistos como espacios en que la dispersión y la presencia de una rica y variada constelación de significaciones e identificaciones les permite a los adolescentes diferenciarse de los demás, posibilitando negociar sus identificaciones de modo de responder a la complejidad y multiplicidad de cada uno como ser pluridimensional. Sin embargo, tal riqueza no impide que en estos escenarios se materialice la agresión y la sanción a la diferencia (Ramírez, 2013).

Así, en el espacio social que constituyen las instituciones de educación secundaria se hacen presentes polifónicos constructos de identificación, posición o localización que se ocupan 
transitoriamente, para desde ahí aparecer frente al Otro en el mundo de la vida, permitiendo al sujeto una perspectiva que le permite apreciar otras representaciones. Sin embargo, es posible visualizar que en estos centros falta mucho todavía para conseguir una implantación más eficaz de las medidas de inclusión y atención a la diversidad (Álvarez et al., 2012).

En este contexto, se comprende que a los estudiantes secundarios se les dificulte asumir la propia singularidad para participar en el mundo de la vida cotidiana, ya que la abrumadora escolarización buscaría, como se plantea en algunos relatos, que sean "uno más”, lo cual daría cuenta de la constitución de un sujeto más en la vida común, anónimo y sin presencia. A lo que se suma la valoración de una tarea pedagógica encaminada a socavar la particularidad. Así, por ejemplo, es posible constatar la existencia de escenarios educativos subsidiarios de la universalidad, donde se sancionan, condenan y reprimen orientaciones no ajustadas a la heteronormatividad.

Particularmente, se aprecia que en las significaciones de género y sexualidad que impregnan las prácticas de la educación sexual en educación secundaria predomina un modelo biomédico que privilegia la heterosexualidad y refuerza los atributos de las masculinidades y feminidades hegemónicas, sin considerar las identidades que se distancian de estos patrones (Venegas, 2011). Lo que conlleva a poner mayor atención al tema del acoso escolar que aparece con la ruptura de los roles de género y a mecanismos como la injuria y el uso de términos peyorativos sobre la masculinidad y la feminidad que buscan conseguir el efecto de delimitar los comportamientos aceptables en este espacio social (Pinos y Pinos, 2011).

Evidenciándose, que en la búsqueda de la inclusión la figura del docente es central, específicamente, considerando que ellos no fueron capacitados en la formación inicial y continua para hacer frente a la diversidad sexual. Poniendo de relieve la necesidad de un esfuerzo colectivo de su parte para acoger a dicha diversidad y sugerir metodologías de enseñanza (Souza, Espinosa, Da Silva y Santos, 2016). Diversidad que escapa a comprensiones constreñidas de ciertos fenómenos que se encapsulan en determinadas definiciones y categorías (Bailey, Vasey, Diamond, Breedlove, Vilain y Epprecht, 2016; Cahill, Baker, Deutsch, Keatley y Makadon, 2016). Un claro ejemplo de esto es la investigación de Blair y Hoskin, quienes concluyeron que las categorías deberían estar en contante de-construcción ya que las que existen son insuficientes y no tienen en cuenta la diversidad sexual y de género de quienes se auto-identifican como lesbianas (2016).

Desde aquí es posible visualizar que los jóvenes que se adscriben a localizaciones no hegemónicas presentan mayor riesgo de victimización y acoso (Bouris, Everett, Heath, Elsaesser y Neilands, 2016; Mullaney, 2016, Pichardo, Molinuevo, Rodríguez y Romero, 2009). Asimismo, debido a que algunos escenarios escolares secundarios se presentan como sistema cerrado, sin ofrecer espacios a las singularidades, solo queda que los que no se ajustan a sus códigos opten por afirmar sus diferencias y su devenir en una especie de auto-apartheid, replegándose a una posición particular o bien recogiéndose en una relación periférica que lucha por algunas trasformaciones, pero dentro del marco de los valores y contenidos políticos y culturales de los sectores dominantes; atisbándose las implicancias de desanudar el sistema cerrado en que se ha ido configurando el saber pedagógico que promueve un sistema escolar totalizador.

En este horizonte se abre la posibilidad que a través de una acción estratégica los sujetosadolescentes que estimen que sus trayectos/proyectos no son viables y sufran 
hostigamiento, puedan y deban modificarlos a través de la acción y el discurso. Lo mismo ocurriría en el proceso de descentramiento sistemático de la normalidad. Desde aquí, las experiencias de los sujetos LGTB nos ofrecen rearticulaciones de las relaciones sociales cotidianas que se generan en estos espacios educativos, abriéndose impensados e impredecibles horizontes conceptuales por sus prácticas transgresoras.

En otras palabras, la disociación universal/particular y las experiencias narradas en los escenarios escolares secundarios desanudan la reflexión en torno a las implicancias de las luchas particulares, en este caso de los sujetos LGTB, quienes han de enfrentar el cariz totalizante de los dispositivos pedagógicos.

Haciendo una analogía con el fenómeno de la ciudad que Eribon (2001) menciona en su libro "Reflexiones sobre la cuestión gay", podemos reconocer que el espacio educativo de la educación secundaria se asemeja al de la gran ciudad, pues entrega la posibilidad de un mayor desarrollo por situarse como una localización polifónica, "un universo de extranjeros", lo que permitiría "preservar el anonimato y por tanto, la libertad", frente a las "trabas sofocantes de las redes de interconocimiento" que se desarrollarían en la escuela primaria, símil a "la vida en las pequeñas ciudades o en los pueblos, donde todo el mundo se conoce y reconoce, y debe ocultar lo que es cuando se aparta de la norma” (pp. 39-40).

\section{Abordaje metodológico}

En consonancia con el desafío de enfrentar el problema de la descontextualización que pervive en las ciencias sociales y humanas, se considera importante la elaboración de un contexto como práctica interpretativa que "trata de seleccionar el escenario más propicio para la historia que deseamos narrar, mismo que es diseñado, además, acorde a nuestra biografía, experiencias, anhelos, tristezas, angustias y culpas, pues ellos marcan la selección de los elementos de la trama” (Contreras, 2012, p. 212). Por lo tanto, se incluyen "algunas categorías muy generales, como Escenario (Tiempo, Lugar), Participantes (y sus varios roles), y un Evento o Acción. [...] Aparte de la comprensión subjetiva de un evento, [...] y un modelo que representa lo que informalmente se llama experiencia” (Van Dijk, 2001, p. 71). Dando cuenta de algunos principios relevantes y particularmente del fenómeno de la ostentación, que remite "al proceso en que el emisor produce un estímulo con la intención de hacer manifiesto o más manifiesto para el oyente un conjunto de supuestos" como un intento de limitar las inferencias y/o guiarla en cierta dirección (Sperber y Wilson, 1994, p. 87).

En este contexto, nos proponemos relevar la experiencia en escenarios escolares secundarios de algunos sujetos adscritos al colectivo LGTB. Para ello se plantea como objetivo descubrir las definiciones que construyen de sus procesos de sujeción, resistencia y visibilización en dichos contextos escolares.

Es importante destacar que la investigación se llevó a cabo en la isla de Gran canaria, la cual constituye una comunidad autónoma de España, con aproximadamente 838.000 habitantes. Debido a su clima y recursos naturales, Gran canaria constituye un importante destino turístico de sol y playa. Al respecto, la isla es considerada por muchos como un "paraíso gay", además que las playas de las "localidades costeras mediterráneas y canarias, sitúan a España entre los países con una mayor cantidad de lugares de referencia gay desde finales del siglo XX” (Fernández, 2007, p. 244). Es en esta misma isla que un grupo de investigadores Españoles estableció que 
un 5\% de los estudiantes se encontraría en una de las categorías de lesbiana, gay, bisexual o persona en cuestionamiento de su identidad sexual. Esto supone que en una clase de 30 estudiantes, hay al menos uno o dos chicos o chicas en esta situación. (Pichardo et al., 2009, p. 77)

De igual manera, es posible mencionar que en el plan de actividades complementarias y extraescolares del gobierno de Canarias se incluye el taller: "Diversidades en construcción: una cuestión de educación", que comenzó durante el curso escolar 2011-2012, manteniéndose hasta la fecha. Su objetivo es contribuir a la tarea de potenciar los valores de igualdad, equidad, justicia y solidaridad, la promoción de entornos inclusivos, así como la prevención de la violencia de género, el sexismo y las LGTB-fobias (Consejería de Educación, Universidades y Sostenibilidad, 2012), evidenciándose por parte de los isleños una clara intención de trabajar en pro de la inclusión de las minorías LGTB en los establecimientos educacionales secundarios.

Hemos de mencionar que esta investigación se desarrolló en el marco del paradigma cualitativo bajo el enfoque fenomenológico, adoptando un diseño de estudio de caso múltiple y retrospectivo, que de acuerdo a Yin (1994) posibilita investigar eventos y puntos de vista particulares, concretos y específicos, dando oportunidad a que se desplieguen experiencias y relatos plurales. De esta forma, se realizaron entrevistas en profundidad a 26 participantes que se adscribían al colectivo LGTB, con edades comprendidas entre 18 y 46 años, y que cursaron sus estudios en establecimientos educacionales de diferentes Ayuntamientos de la Isla de Gran Canaria. Para la selección de los participantes se optó por contactar con el colectivo LGTB Gamá, adicionalmente se invitó a participar a través de un blog, además de conectar con algunas personas en la universidad. La perspectiva se encuadró en y desde un análisis narrativo (Ramírez y Contreras, 2016) en que de acuerdo a lo planteado por Cornejo, Mendoza y Rojas (2008), se privilegia en un primer momento la singularidad de cada experiencia relatada intracaso y en un segundo momento, desde una lógica transversal inter-caso, determinando ejes temáticos-analíticos, realizándose además un análisis estructural (Bernasconi, 2011).

\section{Relatos (hallazgos)}

En este apartado reconstruimos los relatos de los participantes en una trama que intenta desvelar las definiciones que construyen de sus procesos de sujeción, resistencia y visibilización en los contextos escolares, develando los diversos sentidos que han levantado en torno a lo que la educación secundaria les ofrecía. Sin embargo, el enunciar estas tramas no significa que postulemos que ellas son habitadas de forma exclusiva y permanente, solo pretendemos dar cuenta, didácticamente, de las significaciones que se recrean desde un espacio de habitación obligada para los sujetos LGTB. Esto buscaría aportar nuevos elementos a la trama argumental que hace referencia a asentar la premisa que las identificaciones que se configuran en los escenarios escolares son inestables, con altos grados de dispersión o de restricción, ofreciendo o negando identificaciones que articulan diversas experiencias del sí mismo, de tal modo de encumbrar y profundizar posibilidades de dislocación, sabotaje o resistencia.

\subsection{Pluralidad y dispersión}

Esta condición viene a decir que el mundo de la vida es habitado por diversos seres. Dicha pluralidad, que sería condición sine qua non de la existencia humana, se sustenta al ser todos lo mismo, es decir, seres humanos. No obstante, ningún ser es igual a otro, lo que 
imposibilita el fin del mundo común, es decir, impide que el sujeto se vea solo bajo un cariz o se presente "únicamente bajo una perspectiva" (Arendt, 1993, p. 66).

En el colegio, no lo sé... ahora viéndolo desde el punto de vista actual, puedo pensar que había un chico y tal, que se reían de él, pero el resto no, todas eran súper femeninas y todos eran súper masculinos, había como una diferencia muy marcada, ¿no? Nadie se salía de la norma, luego en el Instituto sí, conocí chicos gays, chicas lesbianas, gente diferente, ambiguos y tal...me junte con gente distinta, conocí gente que.... conocí a un chico que era gay, una niña que era bisexual, me dispersé un poquillo, vamos... que encontré ciertos círculos en los que me sentía más cómodo, pero sí es verdad que seguía también siendo objeto de... (Manuel)

El relato de Manuel ilustra que la educación secundaria habría implicado exponerse a una pluralidad que se mantuvo invisible durante la primaria. Invisible porque no tenía nombre, invisible porque el discurso pedagógico en su neutralidad y silencio no posibilitó que apareciera ante los otros. Neutralidad que solo demuestra la homofobia de aquellos que respetaban la norma, “...puedo pensar que había un chico y tal, que se reían de él”, así, el otro en su radical alteridad solo aparece en su condición de rechazado por no ajustarse a lo convenido socialmente, en directa oposición a las predisposiciones que aluden al disciplinamiento del cuerpo, a la construcción de hábitos, movimientos o gestos que la escuela busca que los recién llegados interioricen.

De este modo, el relato de Manuel expresa que la secundaria fue un espacio que le permitió interactuar con una pluralidad, aunque esa pluralidad seguía estigmatizada y siendo centro de agresiones. Al respecto, podríamos presumir que cuando él dice "pero sí es verdad que seguía también siendo objeto de...", el concepto de "objeto" nos remite a una codificación o un distanciamiento de las significaciones constituidas en el hecho de no ajustarse a lo convenido, de ahí que la experiencia de homofobia pase a ser parte del mundo de los objetos. Frente a lo cual surgen dos opciones, la primera, que la codificación ayudó a establecer distancia al apreciar esa experiencia para de esta forma transformarse en crítica, resistencia o sabotaje, y la segunda, que sea un extrañamiento que fragmente al ser.

La pluralidad que Manuel pudo apreciar trajo como efecto un estar disperso, "me dispersé un poquillo”. ¿Qué entendemos por dispersarse? Este término, comúnmente hace referencia al distanciamiento de un valor central, además de un movimiento en diversas direcciones en que al final se pierde la dirección que se había privilegiado al comienzo, y finalmente, alude al desplazamiento para instalarse en una localización un poco alejada del lugar en que inicialmente fue engendrada. A partir de aquí podemos acotar su significación de la siguiente manera: la dispersión viene a recrear una trama en que la heterogeneidad posibilita una afiliación en torno a identificaciones cercanas, lugar en que nuestro entrevistado señala sentirse "más cómodo".

En suma, la pluralidad le permitió a Manuel habitar en espacios con la mayor circulación de identificaciones que pudo negociar, enredándolas con la unidimensionalidad que se le presentaba en primaria, lo que hace con la colaboración de sus pares, por lo que desde esa pluralidad puede vislumbrar modelos que antes no se hallaban a su mano. De ahí que dicha pluralidad, en este relato, materialice la posibilidad de trayectorias de identificación.

\subsection{Respeto a la diferencia}

Aparte que del sistema inglés al español hay un salto también, desde todo en inglés a todo en español, era otra forma de dar clases también, ¿sabes? $\Upsilon$ yo era buena estudiante... me tuve que adaptar al método español, a la libertad, a muchos gays y lesbianas, encontré todo lo que no... Encontré respeto a la diferencia y por esto... no, 
en el colegio no... Los problemas llegaron después, en el bachillerato, cuando pasé del colegio al Instituto, ya llegaron algunos suspensos, faltaba a clases... ya fui más...tenía más libertad. (Carmen)

Otro fenómeno que nos desvelan estos narradores, viene de la mano de Carmen, desde este relato podemos interpretar que la asistencia a la educación secundaria se valora en relación al principio regulador de ser buen estudiante, marcando la diferencia en las causas del desajuste, así la libertad y el encontrar "respeto a la diferencia", serían las condiciones que provocarían que ella no cumpliese a cabalidad su rol de buena estudiante. Con la intención que prevalezca en nuestro análisis la polifonía e incluso las aseveraciones, nos detendremos en la relación de las dos premisas mencionadas, no sin antes admitir que el relato se enreda también en el hecho que la educación secundaria presenta al adolescente una amplia diversidad. Diversidad que en este discurso se ve más cercana a la concepción arendtiana de la presencia de otros que ven y que atestiguan la existencia. Apreciamos que el "respeto a la diferencia", le ofreció a Carmen la posibilidad de escapar de una identidad prefabricada en torno a ser buena estudiante, que representaría lo que previsiblemente triunfa en las sociedades actuales y cuya posición es posible mantener secuestrando al ser. Entonces, la presencia y la diferencia del otro se presenta como una liberación. De ahí que el respeto a dicha diferencia se aprecie al servicio de la apertura a todo un nuevo abanico de prácticas de libertad.

Sin embargo, Carmen consigna en su relato la existencia de un problema, "los problemas llegaron después, en el bachillerato, cuando pasé del colegio al Instituto, ya llegaron algunos suspensos, faltaba a clases...", observamos que tal libertad no se representa en toda su magnitud, pues nuestra entrevistada no sería capaz de escapar totalmente del influjo que el código de buena estudiante ha tenido sobre ella en los escenarios escolares. Desprendiéndose, desde nuestro punto de vista, que la identificación del buen estudiante sería una regulación sistemática "de las conductas de vida en la renuncia, la obediencia, el acatamiento, la sumisión y la esperanza” (Foucault, 1994, p. 23), por lo que transitar por recorridos no definidos de antemano en la escuela, significa un problema para quién se ha formado en este discurso.

De ahí que la libertad y sobre todo, el respeto de la diferencia re-problematizan el código del buen estudiante, desvelando su fragmentación y radical artificialidad.

\footnotetext{
....el Instituto para mí fue una época mucho más bonita que el colegio, sin duda, en muchos aspectos y éste es uno de ellos, de no sentir miedo, de no sentir esa disciplina tan dura y tan limitante, ese control tan estricto, y pasamos de tener 13 a 14 años tampoco había tanta diferencia... pero sí la diferencia era esa. (Oscar)
}

\title{
2.3. Responsabilidad y libertad
}

\begin{abstract}
Si tú te imaginas y tal, en el Instituto tienes que ser responsable, yo creo que son pruebas que ha puesto la sociedad y la cultura de esta sociedad para qué la gente vaya y fracase estrepitosamente, porque responsabilidad y libertad, siendo un adolescente, pues es complicado, entonces, vamos... a lo mejor si fueras un niño espartano y de golpe te metieran en la guerra... yo creo que se estudia más muchas veces en la Universidad que en la época del Instituto, porque estás en la pubertad y tienes otra historia... (Borja)
\end{abstract}

Este relato se distancia de los anteriores pues en él se observa la fuerza del tópico de la pubertad. Este constructo es asociado en el relato de Borja a la imagen del salvaje, del ser que no domina sus instintos. Es decir, surge la idea de un adolescente Dionisíaco, que refiere a un ser individualista, egoísta e impulsivo y que, por lo tanto, debe ser regulado y sometido a una estricta disciplina por parte de los adultos. Esta pubertad a la que se refiere 
nuestro entrevistado, moviliza y evoca una serie de discursos y recursos materiales que buscan intervenir y regular un estado apolíneo, para que el niño no se convierta en un sujeto dionisiaco, aunque para ello deba superar desafíos inalcanzables, como lo significa Borja, "yo creo que son pruebas que ha puesto la sociedad y la cultura de esta sociedad para qué la gente vaya y fracase estrepitosamente", los que nos remite a inferir que este fallo sería intencionalmente ideado, y que respondería a ciertos intereses creados, entre los cuales figura que el fracaso en conjugar responsabilidad y libertad empujaría a los sujetos a encarrilarse en los trayecto predefinidos por los adultos, de manera de alcanzar el éxito en los ámbitos valorados.

Esto respondería también a la premisa que libertad y responsabilidad son dos constructos que no pueden reconciliarse a ciertas edades, llevando a los mismos resultados, es decir, la incapacidad de la infancia para ser protagonistas de su propio desarrollo, obligando a los jóvenes a adecuarse a lo definido por los adultos, desde una perspectiva adultocéntrica.

En este relato, la experiencia de la educación secundaria es significada como un espacio de tensión y conflicto, por intentar tejer los hilos supuestamente destinados a fracasar en una red llamada pubertad. Así, la pubertad es representada por Borja como una narrativa que inunda el escenario escolar secundario, lo que no sabemos bien, es si esa presencia es significada como un acontecer que implica lo imprevisto, lo fluido, lo improbable o más bien fue elaborada desde las representaciones que se han fabricado en torno a ella y que responden a intereses y relaciones de poder, que desde el discurso de Borja se orientan a optar entre participar en el espacio público o recluirse bajo el código de la responsabilidad en la identificación del buen estudiante. Desvelando, además, desde lo enunciado que la responsabilidad en el contexto escolar se ajustaría a los requerimientos de "estudiar".

\subsection{Ser uno más}

En primaria, yo creo que me sentí bastante rechazado, sobre todo en la época en que ya era más grande, como en quinto o sexto y eso sí que creo que fue bastante complicado, en el Instituto no, en el Instituto era uno más, algún comentario como en primaria, pero ya había crecido, estaba más espabilado, era una cosa muy distinta. (Aday)

En este fragmento apreciamos la experiencia de un sujeto que admite la homofobia a la que estuvo expuesto, siendo clave desde nuestro punto de vista el enunciado: "uno más". Desde aquí podemos comprender que quién era el centro del rechazo en un espacio mucho más restringido y homogeneizador, como era la primaria, se definiera en secundaria como "uno más", al tener mayor disponibilidad de espejos.

La visión del instituto como un espacio más amigable para las personas LGTB es compartida también por otro de nuestros entrevistados:

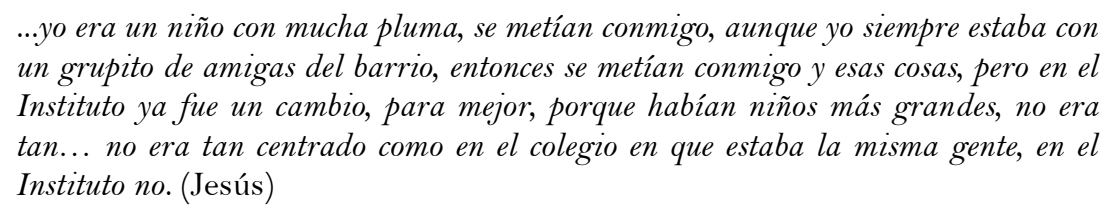

Lo que nos lleva otra vez a la analogía de la ciudad y la escuela secundaria, puesto que en la diversidad que ella envuelve el ser pasa inadvertido, no está frente a los ojos del otro, aludiendo a una presencia impersonal. Desde aquí podemos inferir que la pluralidad otorga la posibilidad de presencia de cada uno desde sus localizaciones, situación que no es privilegio solo de algunos, sino de todos. De este modo, el sujeto puede valorar que es uno más en la vida común y al ser uno más puede participar en ella, puede accionar en la escena 
de sus interpretaciones y significaciones. Así, el mundo de la vida cotidiana no es un "mundo privado", por el contrario, es un "mundo público" que se comparte esencialmente con otros. "Un mundo intersubjetivo que de manera fundamental sostiene una realidad intuitivamente compartida y entendida como válida por todos, como marco común de interpretación” (Estrada, 2000, p. 130).

De ahí que "ser uno más" representa desde las proyecciones recreadas el significante de vida común y participación. “...en el Instituto todo es más impersonal, ya vas más a tu bola, ya hay gente más diversa” (Alba). Probablemente esta diversidad a la que hace referencia la mayoría de nuestros entrevistados, les otorgaría a los sujetos la posibilidad de ir a su "bola", entendiendo el tropo bola como un objeto esférico, de superficie curva que le permite rodar, es decir, traducido al lenguaje de nuestros sujetos, que su vida ruede libremente. Sin embargo, este rodar remite además a un movimiento de retorno al sí mismo, que implica un desplazamiento de este hacía su centro, de ahí que ir "a tu bola" podría aludir también a un retorno.

\subsection{Multi-afiliación}

En el Instituto yo necesitaba sentirme integrado en los grupos, entonces fue cuando hice un esfuerzo e iba cambiando, me juntaba con muchos grupos, quería identificarme, me vestía un año de negro, al año siguiente de... lo que sea... me metí en todas las tribus urbanas buscando mi sitio, ¿no? Pero eso fue, ya te digo, en la etapa ya de mayorcito, en primaria tuvo importancia la ausencia de amigos, sí, sí la tuvo también supongo. (Israel)

El escenario de secundaria sería la oportunidad de poner en juego la flexibilidad, la cual se materializa en múltiples participaciones que se originan desde la pluralidad que se aprecia en estos espacios. Dichas participaciones prometen al joven configurar identificaciones diferenciales que adoptan significados circunstanciales, “... me metí en todas las tribus urbanas buscando mi sitio”, en términos de Bruner (1991), la experiencia de sí o el yo se constituye en las participaciones, mismas que posibilitan "localizar el Yo, no solo en la rapidez de la conciencia privada inmediata, sino también en una situación históricocultural” (p. 109). De este modo, el relato de Israel interpela sobre la importancia de afiliarse, especialmente en la pluralidad de la educación secundaria que es donde está opción se materializa, aunque él admite que por sí sola no basta, requiere además que el ser se exponga, salga al encuentro del otro y lo otro, "hice un esfuerzo e iba cambiando". De esta manera, desde una ética del cuidado y del obrar responsable, podríamos inferir que Israel se valora como héroe, tal como Arendt lo define, "en la voluntad de actuar y hablar, de insertar el propio yo en el mundo y comenzar una historia personal" (1993, p. 201), revelando a un agente. Esta multi-afiliación permite una trayectoria de identificaciones, ofrece modelos y códigos de los que el sujeto se apropia activamente, renegociando y remitiendo a un constante devenir. Sin embargo, cuando Israel anuncia, andaba "buscando mi sitio, ¿no?" pareciera que para él, la identidad no fuera transitoria ni producto de un continuo trabajo, sino más bien, un posición definitiva en la lógica de lograr una meta o alcanzar una certeza. Esto ocurriría solo si definiéramos sitio como sinónimo de identidad. En caso contrario, desde sus diversos significados, sitio se podría asociar más bien a perspectiva u horizonte. Así, un sitio sería una localización, en términos de Braidotti (2002) "un territorio espacio-temporal compartido y construido colectivamente, conjuntamente ocupado" (p. 26), lo que implica que desde un sitio se pueden planear trayectorias. 


\subsection{Violar la ley del silencio}

Yo venía como con mucha rabia acumulada del colegio... entonces me mostraba tal cual era, me daba igual lo que la gente dijera porque me señalaban igual. Hasta que dije, estoy harto ya de tanta cosa, estaba muy reprimido, me daban miedo los profesores, no era capaz de contarle a nadie que tenían un problema, ni siquiera hablaba con mis padres, pero ya en el Instituto sí, o sea era voz populi que yo era el bicho raro del centro. (Aythami)

Desde los diversos relatos la secundaria se constituye en una trama en la que se anuncia la pluralidad, demarcando para algunos sujetos un espacio en que la necesidad, el deseo, el dolor, ya no se puede ni se desea ocultar. De ahí que la educación secundaria no se presente como dique, ni como un espacio en que solo circula una incuestionable interpretación que se negocia en el mundo de la vida. Ya no es una guarida, ni una cárcel de alta seguridad, y por tanto, no ahoga el acontecer educativo. Por lo que, como Aythami expresa, en ella se puede aparecer, se puede tomar la palabra, se puede dejar de disimular. Aquí ya no se le pedirá callarse y ser discreto, puesto que el orden homofóbico se presenta como una más de las relaciones de poder, mientras que en primaria este se aprecia como dominación, entendido como una relación cuyo "margen de libertad es extremadamente limitado", fijo y perdurablemente disimétrico (Foucault, 1994, p. 127). Lo que no impide que igualmente se desencadene la homofobia.

No obstante, nuestro entrevistado en la lógica del cuidado de sí, en un retorno a sí mismo, decide, "estoy harto ya de tanta cosa, estaba muy reprimido, me daban miedo los profesores, no era capaz de contarle a nadie que tenían un problema, ni siquiera hablaba con mis padres". Aythami anuncia una práctica que lo libera parcial y momentáneamente de la coacción que lo amenazaba y le posibilita desde el mostrarse, reiniciar y reencontrar la propia libertad.

En este relato el narrador nos anuncia a un "homosexual que habla de su vida "privada" y rompe la situación "normal", ya que se resiste a la premisa que la homosexualidad no es decible" (Eribon, 2001, p. 149), de esta forma el héroe de esta historia se cuenta como un sujeto que era objeto por la mirada del otro. Desprendiéndose, que la experiencia en la educación secundaria se ve poblada del acontecer, de lo impredecible, de la renovación, de la radical novedad de un ser que ha sido considerado objeto, pero que no obstante, ha asumido que su devenir depende de forma crucial de las relaciones dialógicas con los demás. Puesto que, "ser es ser para otro y a través del otro para mí" (Bajtín, 2000, p. 56).

\section{A modo de conclusión}

Se ha intentado dibujar una cartografía de sujetos que habitan la diferencia, de modo que parecieran ser los únicos diferentes, bombardeados por narrativas de la normalidad, de la homogeneidad que mal entendida ha derivado en rehusar que el ser es diferencia, una radical alteridad.

¿Qué es la normalidad?: nada. ¿Quién es normal?: nadie. Aunque la diferencia hiere, y por eso nuestra primera reacción es negarla. ¿Cómo combatir la imposición de la distinción normalidad-anormalidad?: habitando en el interior de la diferencia, siendo intimo con ella. (Bárcena, 2006, p. 144)

En secundaria un cura me dijo una vez que uno podía tener deseos homosexuales, pero un buen cristiano, un buen hijo de Dios no sucumbía a esos deseos. Él me preguntó: ¿hijo, es que tú no quieres ser normal? (Israel) 
Frente a esta diversidad de panoramas se abren las tramas de la educación secundaria que en su configuración menos restringida multiplica las identificaciones posibilitando la constitución de un mundo común, donde se anuncian las pretensiones de validez que se ponen en escena en el mundo de la vida, validando colectivamente aquello que se ha vuelto cuestionable y permitiendo ser uno más en esa colectividad.

De esta manera, la visibilización del sujeto en el escenario escolar secundario abre curso al encuentro de unos y otros para plantearse hermenéuticamente "la pretensión de que sus emisiones concuerden con el mundo, así como criticar y exhibir los fundamentos de esas pretensiones de validez". Pues el exponerse da pie a reconocer "la reciprocidad en las estructuras del mundo de la vida cotidiana que fijan las formas de la inter-subjetividad del entendimiento y por tanto de la acción posible” (Habermas, 2004, p.179). De modo que participar de forma recíproca significa que se participa en el mismo grado en que se está implicado en ese mundo. Con el mismo grado de exposición, puesto que a todos les acontece algo en la misma medida, experimentándose así contextos singulares de interacción según la propia biografía.

En base a lo anterior, la educación secundaria ofrece a estos narradores, tal vez sin pretenderlo, una oportunidad de no desperdiciar el mundo de la vida. De este modo, parece ser que en la educación primaria las cosas se organizaban arbitrariamente, de manera que nada pasara, y si pasaba era escondido por no ajustarse a lo que los discursos pedagógicos habían ideado. Sin embargo, la educación secundaria con el obrar responsable del sujeto, brinda los enraizamientos basales que constituyen el ámbito esencial de la deconstrucción, para de esta forma potenciar lo vulnerable de la existencia, y con ello ser capaz de hallar los desvíos y fracturas por donde adentrase y perderse, con la complicidad de estar con otros en un mundo que les es propio. Por lo tanto, este espacio se presenta como una apertura para ser un subversivo, resistir o inhibir los efectos de las imposiciones que a fin de cuenta buscan que en el mundo de la vida cotidiana nada ocurra, efecto de la resignación que conllevan los procesos de atomización e individualización del rol de estudiante y sobre todo del "buen estudiante", que debilitan las interacciones e interpretaciones, pero que ofrecen a su vez la opción de un accionar ético (Kjaran y Kristinsdóttir, 2015).

Apreciándose que la escuela secundaria se representa en los relatos como una organización constituida por espacios más inclusivos que los de la primaria, que entregan la posibilidad que cada agente posea un margen de libertad, permitiendo que el sujeto defina estratégicamente como usarla de acuerdo a los propósitos que para él son prioritarios (Foucault, 1994). De igual manera, el sistema educativo de este nivel se constituye en los relatos como una organización no totalitaria, donde sus miembros presencian una pluralidad que ofrece dislocar las categorías jerárquicamente más valoradas, a la vez que no responden con la simple obediencia o el conformismo como ocurre en la educación primaria.

$\mathrm{Al}$ respecto, algunos de nuestros entrevistados en la contingencia de ser descubiertos en su condición de LGTB, iniciaron un proceso de negociación en el que manipularon, controlaron o inventaron formas en función de las circunstancias. Construcciones a veces provisorias y otras permanentes, dentro de un orden de género, sexualidad y afectividad, puesto que habitaban cuerpos en desarrollo que eran re-interpretados y desafiados por nuevas prácticas en que las imágenes eran combatidas, pero también negociadas, ya que los poderes del mundo adulto eran aproximados y al mismo tiempo confrontados, creando diversos caminos (Connell, 1987). 
De igual manera, en educación secundaria es posible apreciar una mayor agencialidad del sujeto en distintas formas y graduaciones, apareciendo un ser humano que se esfuerza por retornar a un obrar responsable y al cuidado de sí mismo, mediante prácticas de resistencia y performatividad en un contexto en que existen escasas identificaciones, mismas que no se condicen con la pluralidad que existe en los territorios escolares. Desde aquí se visualiza la dislocación propia de toda estructura de identidades, así como los reenvíos simbólicos del poder entre los intersticios y los rituales minúsculos de las redes institucionales de los escenarios escolares, que a fin de cuentas son instituciones de secuestro que intentan fijar a los sujetos a la normalización. Es decir, se evidencia una gramática para definir lo masculino y lo femenino, lo que se puede o no desear, aspectos claves en los procesos de significación que estructuran la organización concreta y simbólica de la vida social (Bourdieu, 2008).

Nos referimos a estructuras que se organizan en modelos únicos, unidimensionales y fragmentados de las subjetividades y que sin embargo contienen fisuras, intersticios y desvíos que posibilitan resistencias y performatividades, tal como se aprecia en los relatos construidos por nuestros entrevistados, ofreciendo en ellos, estrategias, apertura y optimismo de retornar a lo vulnerable y finito de la existencia humana, así como a sus acciones, productos y discursos. Desde este optimismo, los sujetos caen en cuenta que todo modelo, sitio, horizonte o morada es transitoria y frágil, pero que es en esa vulnerabilidad donde se hayan las posibilidades de resistir, revertir y renovar, aliviando la pesadez de la existencia. Esta idea que es inicialmente bosquejada por Foucault (1994), enuncia una ética del ser en que la posibilidad de resistencia y transgresión es una práctica moral. De este modo, los fragmentos de relatos reflejan la diversidad, pero sobre todo las prácticas de visibilización, sujeción y resistencia levantadas por nuestros sujetos, donde es posible apreciar como el entorno subjetiviza y construye una experiencia de sí mismo.

\section{Referencias}

Álvarez, V., Rodríguez, A., García, E., Gil, J., López, I., Romero, S., Padilla, M., García, J. y Correa, J. (2012). La atención a la diversidad en los centros de enseñanza secundaria: Estudio descriptivo de la provincia de Sevilla. Revista de Investigación Educativa, 20(1), 225-245.

Arendt, H. (1993). La condición humana. Barcelona: Paidós.

Bailey, J., Vasey, P., Diamond, L., Breedlove, S., Vilain, E. y Epprecht, M. (2016). Sexual orientation, controversy, and science. Psychological Science in the Public Interest, $17(2)$, 45-101. doi:10.1177/1529100616637616

Bajtín, M. (2000). Yo también soy (fragmentos sobre el otro). México: Taurus.

Bárcena, F. (2006). Una diferencia inquietante. Diario de un aprendiz. Teoría de la Educación, 18, 135-152.

Bernasconi, O. (2011). Aproximación narrativa al estudio de fenómenos sociales: principales líneas de desarrollo. Acta Sociológica, 56(4), 9-36

Bernstein, B. (1994). La estructura del conocimiento pedagógico. Madrid: Morata.

Blair, K. y Hoskin, R. (2016). Contemporary understandings of femme identities and related experiences of discrimination. Psychology \& Sexuality,7(2), 101-115 doi:10.1080/19419899.2015.1053824

Bourdieu, P. (2008). El sentido práctico. Buenos Aires: Siglo XXI. 
Bouris, A., Everett, B., Heath, R., Elsaesser, C. y Neilands, T. (2016). Effects of victimization and violence on suicidal ideation and behaviors among sexual minority and heterosexual adolescents. LGBT Health, 3(2), 153-161. doi:10.1089/lgbt.2015.0037

Braidotti, R. (2002). Metamorfosis. Hace una teoría materialista del devenir. Madrid: Akal.

Bruner, J. (1991). Actos de significado. Más allá de la revolución cognitiva. Madrid: Alianza Editorial.

Cadafalch, M. (2001). La educación secundaria ¿Cambio o inmutabilidad? análisis y debate de procesos europeos y latinoamericanos contemporáneos. Revista Española de Educación Comparada, 7, 393-398.

Cahill, S., Baker, K., Deutsch, M., Keatley, J. y Makadon, H. (2016). Inclusion of sexual orientation and gender identity in stage 3 meaningful use guidelines: a huge step forward for LGBT health. LGBT Health, 3(2), 100-102. doi:10.1089/lgbt.2016.0041

Castel, R. (2006). Las metamorfosis de la cuestión social. Una crónica del salariado. Buenos Aires: Paidós

Connell, R. (1987). Gender $\S^{2}$ Power. Stanford, CA: Stanford University Press

Consejería de Educación, Universidades y Sostenibilidad. (2012). Diversidades en construcción: una cuestión de educación. Gobierno de Canarias. Recuperado de http://www.gobiernodecanarias.org/educacion/WebDGOIE/scripts/default.asp? IdSitio $=13 \& \mathrm{IDC}=1571 \&$ cont $=1167$

Contreras, S. (2012). Pedagogía vulnerable y diálogo de saberes. Revista de Investigación Educativa, $3(5), 77-95$

Cornejo, M., Mendoza, F. y Rojas, R. (2008). La investigación con relatos de vida: pistas y opciones del diseño metodológico. Psykhe, 17(1) 29-39. doi:10.4067/s0718-22282008000100004

D'Aloisio, F. (2015). Escuela secundaria como formación personal para la vida ulterior. Dignidad, éxito y ciudadanía. Revista Mexicana de Investigación Educativa, 20(67), 1131-1155.

Eribon, D. (2001). Reflexiones sobre la cuestión gay. Barcelona: Anagrama.

Estrada, M. (2000). La vida y el mundo: distinción conceptual entre mundo de vida y vida cotidiana. Sociológica, 15(43),45-67.

Fernández, V. (2007). Comunidad gay y espacio en España. Boletín de la Asociación de Geógrafos Españoles, 43, 241-260

Foucault, M. (1994). Hermenéutica del sujeto. Madrid: Ediciones de la Piqueta.

Habermas, J. (2004). Teoría de la acción comunicativa I. Madrid: Taurus.

Kjaran, J. y Kristinsdóttir, G. (2015). Queering the environment and caring for the self: Icelandic LGBT students' experience of the upper secondary school. Pedagogy, Culture \& Society, 23(1), 1-20. doi:10.1080/14681366.2014.910250

Pichardo, J., Molinuevo, B., Rodríguez, P. y Romero, M. (2009). Actitudes ante la diversidad sexual. En J. I. Pichardo (Ed.), Adolescentes ante la diversidad sexual. Homofobia en los centros educativos (pp. 70-93). Madrid: Los libros de la Catarata.

Pinos, V. y Pinos, G. (2011). Actitudes de los adolescentes escolarizados de Cuenca hacia la diversidad sexual. Maskana, 2(1), 1-16.

Mullaney, C. (2016). Reshaping time: recommendations for suicide prevention in LBGT populations. Journal of homosexuality, 63(3), 461-465. doi:10.1080/00918369.2016.1124712

Ramírez, M. (2013). La experiencia escolar en las narrativas de identidad sexual LGTB: un estudio fenomenológico retrospective (Tesis doctoral). Universidad Autónoma de Madrid. 
Ramírez, M. y Contreras, S. (2016). Narrativas de identidad afectivo-sexual LGTB en contextos escolares: el aparecer frente al otro. Revista Estudios Pedagógicos, en prensa.

Souza, E., Espinosa, L. Da Silva, J. y Santos, C. (2016). Inclusion of sexual diversity in schools: teachers' conception. REMIE, Multidisciplinary Journal of Educational Research, 6(2), 139152. doi: $10.17583 /$ remie.2016.2004

Sperber, D. y Wilson, D. (1994). La relevancia. Comunicación y procesos cognitivos. Madrid: Visor.

UNICEF. (2010). Educación secundaria. Derecho, inclusión y desarrollo. Desafíos para la educación de los adolescentes. Madrid: UNICEF.

Van Dijk, T. (2001). Algunos principios de una teoría del contexto. ALED, Revista Latinoamericana de Estudios del Discurso, 1(1), 69-81

Venegas, M. (2011). El modelo actual de educación afectivo sexual en España. El caso de Andalucía. Revista Iberoamericana de Educación, 55(3), 1-24.

Vicente, J., Galiano, I. y Miranda, M. (2015). Modelos de formación inicial del profesorado de educación secundaria en España desde una perspectiva europea. Revista Complutense De Educación, 26(3), 741-757. doi:10.5209/rev_rced.2015.v26.n3.44866

Yin, R. (1994). Case study research. Design and methods. Londres: SAGE.

\section{Breve CV de las autoras}

\section{Sylvia Contreras-Salinas}

Doctora en pedagogía de la diversidad sociocultural por la Universidad Complutense de Madrid, Magister en Educación con Mención en Currículo y Evaluación. Socióloga y Profesora de Educación diferencial. Ha trabajado como docente e investigadora en diversas universidades chilenas. Actualmente se desempeña en el Departamento de Educación de la Universidad de Santiago de Chile. En 2015 se adjudica un proyecto Fondecyt de Iniciación titulado: Trayectorias educativas y saberes socialmente producidos que despliegan mujeres inmigrantes en los procesos de inclusión en la Región Metropolitana de Chile. orcid.org/0000-0003-2297-2399. Email: sylvia.contreras.s@usach.cl

\section{Mónica Ramírez-Pavelic}

Docente e investigadora. Doctora en Psicología y Educación por la Universidad Autónoma de Madrid. Psicóloga y Profesora de Educación diferencial. Se ha desempeñado en diversas Universidades Chilenas. Actualmente trabaja como evaluadora en la Agencia de la Calidad de la Educación del Gobierno de Chile. orcid.org/0000-0003-2710-2982. Email: trapecio@gmail.com 